Abstract 269 Table 1 Associations of ITGAM SNPs with SLE subphenotypes \& auto-antibodies ( $n=248$ )

\begin{tabular}{lccccccc} 
SNP & Allele & $\begin{array}{c}\text { Lupus nephritis } \\
\text { OR }(95 \% \text { Cl) }\end{array}$ & $p$ & $\begin{array}{c}\text { Discoid rash } \\
\text { OR (95\% Cl) }\end{array}$ & $p$ & $\begin{array}{c}\text { Anti-Sm } \\
\text { OR (95\% Cl) }\end{array}$ & $p$ \\
\hline rs4561481 & G & $1.39(1.08-1.78)$ & 0.009 & $0.58(0.36-0.91)$ & 0.03 & $1.54(0.96-2.47)$ & 0.07 \\
rs8051304 & C & $1.39(1.08-1.78)$ & 0.009 & $0.58(0.36-0.91)$ & 0.03 & $1.54(0.96-2.47)$ & 0.07 \\
rs889551 & A & $1.39(1.09-1.77)$ & 0.008 & $0.57(0.36-0.91)$ & 0.03 & $1.65(1.02-2.65)$ & 0.04 \\
rs4889640 & C & $1.39(1.08-1.78)$ & 0.009 & $0.58(0.36-0.91)$ & 0.03 & $1.54(0.96-2.47)$ & 0.07 \\
rs889549 & C & $1.39(1.08-1.78)$ & 0.009 & $0.58(0.36-0.91)$ & 0.03 & $1.54(0.96-2.47)$ & 0.07 \\
rs11645526 & A & $1.39(1.08-1.78)$ & 0.009 & $0.58(0.36-0.91)$ & 0.03 & $1.54(0.96-2.47)$ & 0.07 \\
rs8057320 & C & $1.39(1.08-1.78)$ & 0.009 & $0.58(0.36-0.91)$ & 0.03 & $1.54(0.96-2.47)$ & 0.07 \\
rs7193943 & G & $1.39(1.08-1.78)$ & 0.009 & $0.58(0.36-0.91)$ & 0.03 & $1.54(0.96-2.47)$ & 0.07 \\
rs11865830 & G & $1.39(1.08-1.78)$ & 0.009 & $0.58(0.36-0.91)$ & 0.03 & $1.54(0.96-2.47)$ & 0.07 \\
rs3764327 & T & $1.39(1.08-1.78)$ & 0.009 & $0.58(0.36-0.91)$ & 0.03 & $1.54(0.96-2.47)$ & 0.07 \\
rs7196256 & T & $1.41(1.10-1.81)$ & 0.006 & $0.57(0.36-0.90)$ & 0.02 & $1.51(0.94-2.43)$ & 0.10 \\
rs3815801 & C & $1.39(1.08-1.78)$ & 0.009 & $0.58(0.36-0.91)$ & 0.03 & $1.43(0.90-2.28)$ & 0.14 \\
rs2359661 & A & $1.46(1.13-1.89)$ & 0.002 & $0.59(0.37-0.93)$ & 0.03 & $1.36(0.85-2.16)$ & 0.23 \\
rs1143679 & A & $1.67(1.34-2.08)$ & 0.03 & $0.39(0.06-2.58)$ & 0.46 & $0.35(0.05-2.28)$ & 0.30
\end{tabular}

(uncorrected p-values shown)

that a mutation at 5'UTR region of TLR2 gene could be associated with susceptibility/resistance to SLE and malaria. We performed a hospital based case-control study on SLE patients residing in $P$. falciparum endemic areas.

Methods Two hundred female SLE patients and age and sex, matched healthy controls were enrolled. 120 P. falciparum infected patients including 50 uncomplicated cases and 70 severe malarial patients were included.. TLR2 (23bp ins/del) polymorphism was typed by polymerase chain reaction (PCR). $20 \%$ samples were randomly sequenced for validation of PCR results.

Results The mean age and disease duration of SLE patients were 27.44 and 2.91 years respectively. Prevalence of mutants (ins/del+del/del) of TLR2 gene polymorphism were significantly lower in SLE patients compared to healthy controls $(p=0.02 ; O R=0.54)$. Distribution of TLR2 variants were comparable among different clinical phenotypes of SLE. The TLR2 5'UTR mutants were associated with elevated TNF alpha, IL1 beta and IL6 compared to the wild genotypes. Mutants were more prevalent in severe malaria patients than uncomplicated cases $(\mathrm{p}=0.05$; OR=2.31).

Conclusions TLR2 5'UTR 23bp ins/del variants are associated with development of severe disease in $P$. falciparum malaria but possibly an evolutionary mechanism to protect SLE patients against severe malaria in endemic areas

\section{CIRCULATING TREGS AND TH17 CELLS PERCANTEGES IN CLASS IV DIFFER FROM OTHER CLASSES OF LUPUS NEPHRITIS}

${ }^{1} \mathrm{~B}$ Foroncewicz* ${ }^{2} \mathrm{~K}$ Bocian, ${ }^{1,3} \mathrm{~K}$ Mucha, ${ }^{1} \mathrm{~A}$ Wirkowska, ${ }^{1,4} \mathrm{~T}$ Anna, ${ }^{5} \mathrm{~A}$ PerkowskaPtasińska, ${ }^{2,6} \mathrm{G}$ Korczak-Kowalska, ${ }^{1,3} \mathrm{~L}$ Paczek. ${ }^{1}$ Medical University of Warsaw, Immunology- Transplantology and Internal Diseases, Warszawa, Poland; ${ }^{2}$ Institute of Zoology- Faculty of Biology- University of Warsaw, Immunology, Warsaw, Poland; ${ }^{3}$ Polish Academy of Sciences- Warsaw, Institute of Biochemistry and Biophysics, Warsaw, Poland; ${ }^{4}$ Medical University of Warsaw, Postgraduate School of Molecular Medicine, Warszawa, Poland; ${ }^{5}$ Medical University of Warsaw, Transplantation Medicine- Nephrology and Internal Diseases, Warszawa, Poland; ${ }^{6}$ Medical University of Warsaw, Clinical Immunology, Warszawa, Poland

\subsection{6/lupus-2017-000215.271}

Background and aims Lupus nephritis (LN) is a severe complication of systemic lupus erythematosus. T lymphocytes with regulatory properties (Tregs) play a role in preventing autoimmunity, are involved in LN pathogenesis and may also determine glomerular lesions in LN. Their potential use as LN biomarkers is investigated.

The aim of our study was to assess the relationship between repeated measurements of Tregs proportions, histopathology classes and five-year clinical outcomes in LN patients with different disease duration and activity.

Methods Forty eight LN patients were followed-up for 5 years. Their mean age, disease duration and activity (SLEDAI) at baseline was 41.1 years, 9.8 years and 8.3 points, respectively. Their blood was collected twice: at baseline and after 6 months. Populations of Tregs and Th17 cells were analysed by 
flow cytometry, in relation to clinical parameters and previously established LN classes assessed according to the ISN/RPS 2003 classification.

Results Lymphocytes percentages in class IV were different from classes III, V or a combination of III and V. In the latter classes, the percentages of the Tregs and Th17 cells were significantly lower, whereas in class IV the increase in FOXP3 in the Tregs and Th17 cells over six months period was significantly higher (Table 1). Changes in glomerular filtration rate and SLEDAI within 5 years did not correlate with single or repeated Tregs measurements.

Conclusions Differences in lymphocyte proportions between class IV and other classes may suggest its distinct pathogenesis and warrants further investigations on their role as $\mathrm{LN}$ biomarker.

\section{CLINICAL SIGNIFICANCE OF ANTI-DNA/NR2 ANTIBODIES IN DE NOVO NPSLE AND POST-STEROID NPSLE}

${ }^{1} \mathrm{Y}$ Fujieda*, ${ }^{2} \mathrm{~S}$ Mader, ${ }^{2} \mathrm{~V}$ Jegnathan, ${ }^{2} \mathrm{Y}$ Arimuna, ${ }^{1} \mathrm{Y}$ Shimizu, ${ }^{1} \mathrm{M}$ Kato, ${ }^{1} \mathrm{~K}$ Oku, ${ }^{1} \mathrm{~T}$ Bohgaki, ${ }^{1} \mathrm{O}$ Amengual, ${ }^{1} \mathrm{~S}$ Yasuda, ${ }^{2} \mathrm{~B}$ Diamond, ${ }^{1} \mathrm{~T}$ Atsumi. ${ }^{1}$ Hokkaido University Graduate School of Medicine, Division of Rheumatology- Endocrinology and Nephrology, Sapporo, Japan; ${ }^{2}$ The Feinstein Institute for Medical Research, Centre of Autoimmune and Musculoskeltal Diseases, New York, USA

10.1136/lupus-2017-000215.272

Background and aims Anti-DNA/NR2 antibodies are a subset of anti DNA autoantibodies that cross-react with the extracellular domain of the GluN2A/GluN2B subunits of the $\mathrm{N}$ methyl-d-aspartate receptor 2 (NR2), which induce apoptosis of hippocampus neurons and psychiatric disorder in mice and humans. Neuropsychiatric SLE (NPSLE) can develop after initiation of steroid (post-steroid neuropsychiatric manifestation: PSNP) or before treatment (de novo NPSLE). The objective of this study was to clarify the prevalence of anti-DNA/NR2 antibodies in PSNP-SLE and de novo NPSLE

Methods This study involved a cohort of patients with NPSLE who were admitted to Hokkaido University Hospital. NPSLE patients were classified into two groups, de novo NPSLE and PSNP-SLE. Serum anti-DNA antibodies and anti-DNA/NR2 antibodies were measured using in-house ELISAs.

Results Serum samples were obtained from 29 patients with de novo NPSLE, 26 with PSNP-SLE and 83 healthy controls (HC). The levels of anti-DNA antibodies in patients with de novo NPSLE and PSNP-SLE were significantly higher than those in healthy controls (de novo NPSLE, PSNP-SLE, HC: $1.34 \pm 0.09,1.40 \pm 0.14,0.33 \pm 0.03, \mathrm{p}<0.0001)$. The levels of anti-DNA/NR2 antibodies were highest in de novo NPSLE and in PSNP-SLE and HC (de novo NPSLE, PSNP-SLE, HC: 0.75 $\pm 0.10,0.60 \pm 0.07,0.49 \pm 0.03)$. In PSNP-SLE, the frequency of mood disorders was higher than that in de novo NPSLE (58\% vs $31 \% \mathrm{p}<0.05)$.

Conclusions The levels of anti-DNA/NR2 in PSNP-SLE are lower than in de novo NPSLE, indicating the differences in the pathogenesis of these two conditions.

\section{3 URINARY TWEAK LEVELS AS BIOMARKER OF LUPUS NEPHRITIS IN COLOMBIAN SLE PATIENTS}

'BL Ortiz Reyes, ${ }^{1} \mathrm{~T}$ Urrego, ${ }^{2,3} \mathrm{AL}$ Vanegas, ${ }^{4} \mathrm{G}$ Aroca, ${ }^{4} \mathrm{~A}$ Cadena Bonfanti, ${ }^{4} \mathrm{~L}$ Almendrales, ${ }^{2,3} \mathrm{CH}$ Muñoz, ${ }^{2} \mathrm{LA}$ González, ${ }^{4,5} \mathrm{~A}$ Iglesias, ${ }^{1,2} \mathrm{G}$ Vásquez, ${ }^{1,2} \mathrm{JA}$ Gómez-Puerta*. ${ }^{1}$ Universidad de Antioquia, Grupo de Inmunología Celular e Inmunogenética GICIC, Medellín, Colombia; ${ }^{2}$ Universidad de Antioquia, Grupo de Reumatología, Medellin, Colombia; ${ }^{3}$ Hospital San Vicente Fundación, Rheumatology, Medellin, Colombia; ${ }^{4}$ Universidad Simón Bolivar, Grupo investigación Nefrología, Barranquilla, Colombia; ${ }^{5}$ Universidad Nacional de Colombia, Facultad de Medicina, Bogotá, Colombia

\subsection{6/lupus-2017-000215.273}

Background and aims TNF-like WEAK inducer of apoptosis (TWEAK), a TNF ligand superfamily is mainly produced by monocytes/macrophages, and is widely expressed at the RNA level in tissues including kidneys. The usefulness of urinary TWEAK (uTWEAK) to identify renal involvement in Mestizo and African-Latin American (ALA) SLE patients has not been examined yet.

Methods Patients meeting the revised ACR criteria for SLE were recruited from 2 different centres at Medellín and Baranquilla, Colombia. uTWEAK were measured using an ELISA kit ( $\mathrm{R}$ and $\mathrm{D}$ system, USA)

Results 158 SLE patients were recruited (89\% female) with median age of $32.8 \pm 12.1$ years and median disease duration of 7.27 \pm 6.6 years. Mestizo (77\%) and ALA (20\%) were majority. $64 \%$ of patients had lupus nephritis (LN). 50 out of 71 biopsy proven LN had proliferative forms. Mean SLEDAI score was $8.5 \pm 8.7$. LN patients $(2803 \pm 6086$ vs $672 \pm 1042$, $\mathrm{p}=0.013) \quad($ Fig 1A) and ALA patients (3995 \pm 9656 vs 1618 $\pm 2653, p=0.002$ ) had significant higher levels of uTWEAK. uTWEAK levels were higher in patients with active LN and in Class V LN (Fig. 1B). uTWEAK levels were significantly correlated with 24 hours proteinuria, SLEDAI (Fig. 1C) and serum anti-C1q titers. An ROC curve constructed showed a good level of sensitivity and specificity (Fig. 1D)

Conclusions In our cohort of Colombian SLE patients, uTWEAK levels were 4 and 2 times higher in LN patients and ALA respectively. UTWEAK were significantly higher in active LN and were correlated with disease activity, proteinuria and anti-C1q antibodies.

\section{PREVALENCE OF ANTI-DFS70 ANTIBODIES IN A COLOMBIAN COHORT: A CASE-CONTROL STUDY}

${ }^{1} \mathrm{~S}$ Arteaga, ${ }^{2} \mathrm{~T}$ Urrego, ${ }^{3} \mathrm{LM}$ Peñaranda, ${ }^{4} \mathrm{~A}$ Londoño, ${ }^{4} \mathrm{ML}$ Cardenas, ${ }^{1} \mathrm{AL}$ Vanegas, $\mathrm{CH}$ Muñoz ${ }^{1}{ }^{1} \mathrm{M}$ Escobar, ${ }^{1} \mathrm{~W}$ Rojas, ${ }^{1,2} \mathrm{G}$ Vásquez, ${ }^{1} \mathrm{LA}$ González, ${ }^{3} \mathrm{C}$ Perez Koller, 1,2,4JA Gómez-Puerta*. 'Universidad de Antioquia, Sección de Reumatología, Medellin, Colombia; ${ }^{2}$ Universidad de Antioquia, Grupo de Inmunología Celular e Inmunogenética, Medellin, Colombia; ${ }^{3}$ Werfen, Inova Diagnostics, Bogotá, Colombia; ${ }^{4}$ Dinamica IPS, Autoinmunidad, Medellin, Colombia

\subsection{6/lupus-2017-000215.274}

Background and aims Anti-dense fine speckled 70 (anti-DFS70) antibodies were initially identified as an ANA IIF pattern from patients with interstitial cystitis; however, some recent studies showed that anti-DFS70 antibodies are common among ANA 